\title{
COMPORTEMENT DE L'ESPËCE "SALMO TRUTTA" DANS LE BASSIN DE LA SEINE
}

\author{
par J. ARRIGNON
}

Ingénieur agricole I. A. N.,

Chargé de la $1^{\text {re }}$ Région Piscicole au Conseil Supérieur de la Pêche.

\section{SOMMAIRH}

Depuis un certain nombre d'années, on observe, en Basse Seine, au printemps, des rassemblements de Salmonidés. Ces rassemblements concernent des sujets non adultes. Par ailleurs, un certain nombre d'individus adultes, marquís en Ile-de-France en 1961-1962-1963, ont été capturés très en aval de leur point de déversement. Etant donné les investissements supportés par les pêcheurs pour repeupler les cours d'eau tant en truites immédiatement pêchables qu'en alevins et truitelles, il devenait nécessaire d'essayer de comprendre les raisons des dévalaisons constatées et d'en chiffrer l'importance.

Ces investigations ont eu recours à un marquage de poissons, systématique et organisé sur l'ensemble de quatre grandes régions hydrographiques du fleuve Seine :

- Bassin de la Marne Supérieure;

- Bassins de la Seine et de l'Aube Supérieures;

- Bassin de l'Aisne Supérieure:

- Ile-de-France.

Au 31 octobre 1967, sur 30.030 sujets marqués, 3.138 ont été capturés et signalés.

Sur ces 3.138 sujets, 3.049 n'avaient pas quitté le bassin dans lequel ils avaient été déversés.

Le pourcentage général des retours de marques atteint $10,15 \%$ et celui des retours lointains : $3,06 \%$ oo.

Le pourcentage de retours le plus élevé est celui du Bassin de l'Aisne Supérieure où, localement, $34,80 \%$ des sujets marqués ont été repris. Mais, on notera un taux de dévalaison en Basse Seine et en mer de 2,94\% pour la Marne Superieure, $2,20 \%$ oo pour l'Aube et la Seine Supérieures et $4,71 \%$ oo pour l' Ile-de-France.

Le déplacement record est celui d'une truite déversée dans les Ardennes et qui a été capturée à Dunkerque après un voyage de $805 \mathrm{~km}$ dont 530 en eaı douce.

Ces investigations par marquage ont été complétées par des études biométriques faites sur des échantillons prélevés en Basse Seine lors des grands rassemblements de fin d'hiver.

Un certain nombre de constatations en découlent : à dévaler.

- Il y a des lots de poissons qui, plus que d'autres, ont des tendances

- Les souches exotiques péninsulaires (danoises) fournissent un taux plus élevé de migrateurs que les souches indigènes continentales.

- Certains sujets ont des déplacements irréguliers soit vers l'amont, soit vers l'aval, à la recherche d'un milieu plus favorable. 

convenables.

- Les reproducteurs migrent plus ou moins loin à la recherche de frayères

- Les populations locales sont très sédentaires si elles vivent dans un milieu stable et inviolé.

- Les rassemblements en Basse Seine comportent, sous une livrée identiquement argentée, des truites aussi bien fario qu'arc-en-ciel, de même que des Salmo salar.

- Mais il n'est pas possible d'effectuer une discrimination entre Salmo trutta fario (dulcaquicola) et trutta fario (marina) : les sujets migrateurs de l'espèce changent de robe lors de la motivation physiologique et l'on retrouve alors tous les intermédiaires possibles entre truite de rivière et truite de mer.

\section{I. - INTRODUCTION}

\section{P L A N}

II. - TECHNIQUES ET MÉTHODES

2.0. - OBJECTIFS.

2.1. - Organisation des investigations.

2.2. - MARQUAGE.

2.3. - ECHANTILlonNages.

III. - RÉSUltats DE LA CAMPAGNE DE MARQUAGE

3.0. - IMPortance DES MARQUages.

3.1. - Importance des retours de marques.

3.2. - Déplacements.

32.0. - Les grands itinérants eurhyalins.

32.1. - Les concentrations en basse Seine.

32.2. - Les déplacements potamiques acycliques.

3.3. - SÉDENTARISMF

33.0. - Chez les races pures.

33.1. - Après enrichissement en sujets exotiques.

IV. - EXAMEN DES ÉCHANTILLONS

4.0. - MÉThodes.

4.1. - Données DE base.

4.2. - Répartition Des tailles.

4.3. - Poids, engraissement et SExe.

4.4. - Rapponts biométriques.

44.0. - Rapports $\frac{\mathrm{L}}{\mathrm{T}}$ et $\frac{\mathrm{L}}{\mathrm{P}}$.

44.1. - Rapports $\frac{\mathrm{L}}{\mathrm{II}}$ et $\frac{\mathrm{L}}{d}$.

44.2. - Formules vertébrale et scalaire. Écart scalaire Vomer.

4.5. - Aspect général.

45.0. - Nageoires.

45.1. - Robe.

V. - CONCI.USIONS

5.0. - ERREurs et difficultés D'APpréciation.

50.0. - Erreurs opératoires.

50.1. - Erreurs de mesure.

50.2 . - Difficultés d'appréciation.

50.3. - Difficultés d'interprétation.

5.1. - Quelques constatations finales.

VI. - BIBLIOGRAPHIE 


\section{I. - INTRODUGTION}

Les spécialistes qui se sont penchés sur la systématique des Salmonidés ont toujours eu des difficultés à présenter une classification qui satisfasse pleinement un esprit rigoureux. La dernière en date (12) qui distribue dans le sous-ordre des Salmonoedei, les familles des Salangidae, des Osmeridae, des Thymallidae, des Coregonidae, des Plecoglossidae et des Salmonidae, n'apporte aucun jour nouvcau par exemple sur la position, dans cette dernière famille et dans le genre Salmo, de la truite que l'on nomme communément "Truite de mer".

Quand le Docteur Derrion (7) écrit que parler de la "Truite de mer ", c'est ouvrir " la bouteille à l'encre ", il exprime une opinion partagée par bien des personnalités qui se penchent sur la discrimination des truites itinérantes que l'on trouve, non seulement dans les fleuves côtiers de la Manche, mais bien plus encore dans le Bassin de la Seine.

La confusion qui entoure cette discrimination est fort ancienne puisqu'on la trouve dès le Iv ${ }^{\mathrm{e}}$ siècle dans les vers d'Ausone (5) alors que dans son poème La Moselle il décrit quatorze espèces de poissons dont, semble-t-il, fort curieusement trois Salmonidés :

Purpureis que Salar stellatus tergo guttis

"I a Salar, dont la peau est constellée de tàches rouges"

Nec te punices rutilantem viscere Salmo transierim.

« Et je ne t'oublicrai pas, Saumon, tout rutilant de ta chair pourprée ».

Teque, inter species geminas, neutrumque et utrumque,

Qui necdum Salmo, nec jam Salar, ambiguusque, Amborum medio Fario intercepte sub oevo?

«Et toi, Fario, qui es, entre deux espèces jumelles, ni l'une ni l'autre et cependant l'unc et l'autre,

Qui n'est plus truite et pas encore saumon, être ambigu, arrêté au milieu de sa vie? "

La "Salar " serait truite, le "Salmo " saumon et la " Fario " ni l'une ni l'autre.

Depuis lors, diverses introductions connues et bien davantage occultes sont venues ajouter à la confusion, qu'il s'agisse de races de truites fario venant de bassins fluviaux parfois fort éloignés ou de certaines variétés de truites arc-en-ciel importées d'Outre-Atlantique, voire même de déversements dans un même cours d'eau de sujets des nombreuses races de l'une et l'autre espèces.

Les rassemblements de salmonidés observés en Basse Seine chaque année en mars-avril en donnent la meilleure preuve. Lors d'un sondage 
effectué par nos soins le 25 avril 1965 à l'embouchure de l'Austreberthe, petit tributaire de la Seine entre Rouen et Le Havre (1), les 63 sujets pêchés à l'électricité constituaient un véritable "cocktail " comprenant :

4 truites fario d'élevage récent ;

4 truites arc-en-ciel, donc d'élevage ;

23 truites à robe fario ordinaire;

26 truites argentées, ayant les caractéristiques des truites de mer et 6 sujets ayant tout à fait celles de jeunes saumons.

Les observations de même ordre se répètent d'année en année. Elles ont conduit à organiser et à généraliser les investigations par marquage de poissons dans le cours supérieur des grands affluents et sousaffluents de la Seine ainsi que dans les zones de grande activité halieutique : Ile-de-France, notamment.

Associées à des mesures biométriques, elles ont permis d'appréhender la fluctuance des caractères de salmo trutta, mais il est bien certain qu'au stade de la différenciation variétale - s'il s'agit de différenciation variétale, ce qui n'est pas sùr lorsque l'on spécule sur des phénomènes migratoires - seules les techniques de recherche les plus récentes en matière de physiologie, de biochimie, d'endocrinologie, d'embryologie, de génétique ct autres, permettront de donner les bases, non pas d'une discrimination entre Salmo Salar et Salmo Trutta dite marina, mais d'une discrimination entre Salmo Trutta fario et Salmo Trutta dite marina.

Certes l'intérêt de telles investigations ne fut pas d'ordre scientifique à l'origine, mais très naturellement d'ordre technique et financier. Technique, car il importait de déterminer dans quelle mesure, en introduisant d'une façon très anarchique des quantités mal connues de truites d'origine réelle inconnue, se trouvant à l'occasion dans un état sanitaire douteux, on perturbait le milieu aquatique récepteur. Financier, car il importait tout autant de cerner la part utile d'un investissement annuel, connu, d'un montant de un million de francs, consacré, non seulement aux déversements surdensitaires de truites destinées à être pêchées dans les délais les plus courts, mais également aux repeuplements de fond, avec un nombre considérable d'alevins et de truitelles.

Ces raisons amenèrent les Fédérations Départementales d'Associations de Pèche et de Pisciculture de l'ancienne Seine-et-Oise et de l'Oise, des Ardennes, de la Haute-Marne et de l'Aube, à se préoccuper du devenir des truites introduites chaque année dans leurs eaux et à consacrer des sommes importantes au marquage d'une part de leurs enrichissements.

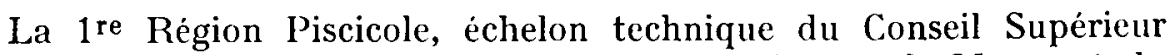
de la Pêche, couvrant alors les Bassins de la Seine, de le Meuse et du Rhin, eût toutes possibilités pour organiser, coordonner et exploiter ces investigations et les assortir de sondages, notamment en Basse Scine. 


\section{II. - TEGHNIQUES ET MÉTHODES}

\section{0. - OBJECTIFS}

Une première opération générale en Seine-et-Oise, de marquage sur des lots importants de truites offrant une taille légale de capture, provenant d'élevage et destinées à des enrichissements massifs de cours d'eau très pêchés, a montré en 1962, par la localisation des recaptures, que de nombreux sujets marqués étaient pèchés très en aval de leur point de déversement et parfois à toute proximité de l'estuaire de la Seine.

Les rassemblements de salmonidés en Basse Seine, depuis longtemps signalés par les riverains, ont été sondés tant par pêche à la ligne organisée (Fédération Départementale des A. P. P. de Seine-Maritime) que par pêche électrique (1 ${ }^{\text {re }}$ Région Piscicole) : les sujets capturés avaient rarement la taille légale de capture et ne provenaient pas d'enrichissements de fond effectués localement, la Seine étant polluée d'une façon chronique à partir de Rouen.

Les objectifs se dégagèrent d'eux-mèmes des constatations effectuées ci-avant :

- Il y a dévalaison importante de truites dans le Bassin de la Seine.

- Quelle est l'importance de ces dévalaisons ?

- Quand il y a dévalaison de poissons destinés aux enrichissements :

- Quelle est la part des sujets sédentaires?

- Et celle des sujets itinérants?

- S'agit-il de migrations ou de déplacements?

- S’il s'agit de déplacements :

- Quelle est la part du milieu?

- Quelle est la part de l'environnement?

- Quelle est la part de l'individu, dans la motivation de ces déplacements?

- S'il s'agit de migrations :

- Quelles sont les origines des lots comportant un important contingent de migrateurs, animés par un déterminisme génétique certain, se traduisant par les modifications morphologiques et physiologiques que l'on connaît?

Et enfin :

- Quelles directives d'enrichissement piscicole et quelles propositions d'aménagement du milieu aquatique peuvent, s'il y a lieu, limiter les phénomènes en cause aux taux de dévalaison ordinairement observés sur les cheptels de truites dites indigènes?

\section{1. - ORGANISATION DES INVESTIGATIONS}

Comme dit plus avant, l'appréciation de tels phénomènes ne pouvait résulter que du marquage systématique de quantités très importantes de truites et supposait des efforts d'organisation, de coordination et 
d'exploitation que seule peut réaliser une entité technique couvrant les grandes unités hydrographiques que sont les bassins fluviaux et ayant au surplus des relations étroites avec les collectivités piscicoles exploitant ces bassins, et d'autre part, avec les organismes scientifiques et techniques s'occupant de pêches maritimes et ayant eux-mêmes d'étroites relations avec les gens de mer. L'accent doit être mis sur ces relations qui conditionnent absolument la crédibilité des résultats statistiques de telles investigations.

En bref, en ce qui concerne le Bassin de la Seine, l'organisation a compris, jusqu'au 31 octobre 1967 :

- La planification des opérations de marquage dans les têtes de bassin des grands affluents et sous-affluents.

- L'exploitation d'un fichier régional comprenant tous les renseignements ayant trait aux opérations sus-visées.

- L'harmonisation des modes opératoires et la standardisation du matériel employé.

- Une information judicieuse des pêcheurs et l'octroi de récompenses à ceux qui fournissent le renseignement.

- Le collationnement de ces renseignements et une tenue rigoureuse d'un fichier dynamique d'exploitation des renseignements.

L'articulation du Fichier Régional de marquage a été donné par ailleurs (2).

Toutes les collectivités pisciccles et organismes ayant à voir avec la pêche et la navigation tant fluviales que maritimes ont été périodiquement informés soit directement, soit à l'occasion des opérations par voie de presse, de radio et de télévision, de la part qu'elles pouvaient prendre au succès de cette investigation en fournissant tous renseignements sur les captures éventuelles de sujets marqués, ou encore en envoyant au laboratoire de la Région Piscicole des échantillons complets destinés aux études biométriques, ou à défaut d'échantillons, des écailles de ces échantillons pour lectures scalimétriques.

Les marquages proprement dit ont été opérés :

- En démonstration par les techniciens de la Région Piscicole;

- En opérations générales et systématiques par les Brigades de Gardes Pêche des Fédérations Départementales intéressées, soit :

- La Fédération Départementale des A. P. P. des Ardennes pour le Bassin de l'Aisne Supérieure ;

- La Fédération Départementale des A. P. P. de la Haute-Marne pour les Bassins de la Marne et de l'Aube Supérieures;

- La Fédération Départementale des A. P. P. de l'Aube pour le Bassin de l'Aube et celui de la Seine Moyenne;

- Les Fédérations Départementales de la Seine-et-Oise, de l'Oise et de la Seine-et-Marne pour les tributaires de la Seine en Ile-de-France.

Les échantillonnages par pêche électrique et les études biométriques ont été accomplis par le personnel de la $1^{\text {re }}$ Région Piscicole mais, comme déjà dit ci-avant, un nombre important d'échantillons fut envoyé par des expéditeurs bénévoles que l'on ne saurait trop remercier. 


\section{2. - OPÉRATIONS DE MARQUAGE}

Le marquage fut opéré, dans la grande majorité des cas, par sertissage d'une agrafe autour du maxillaire inférieur du poisson. Cette agrafe est faite d'un métal inoxydable et porte, gravés, deux lettres et quatre chiffres.

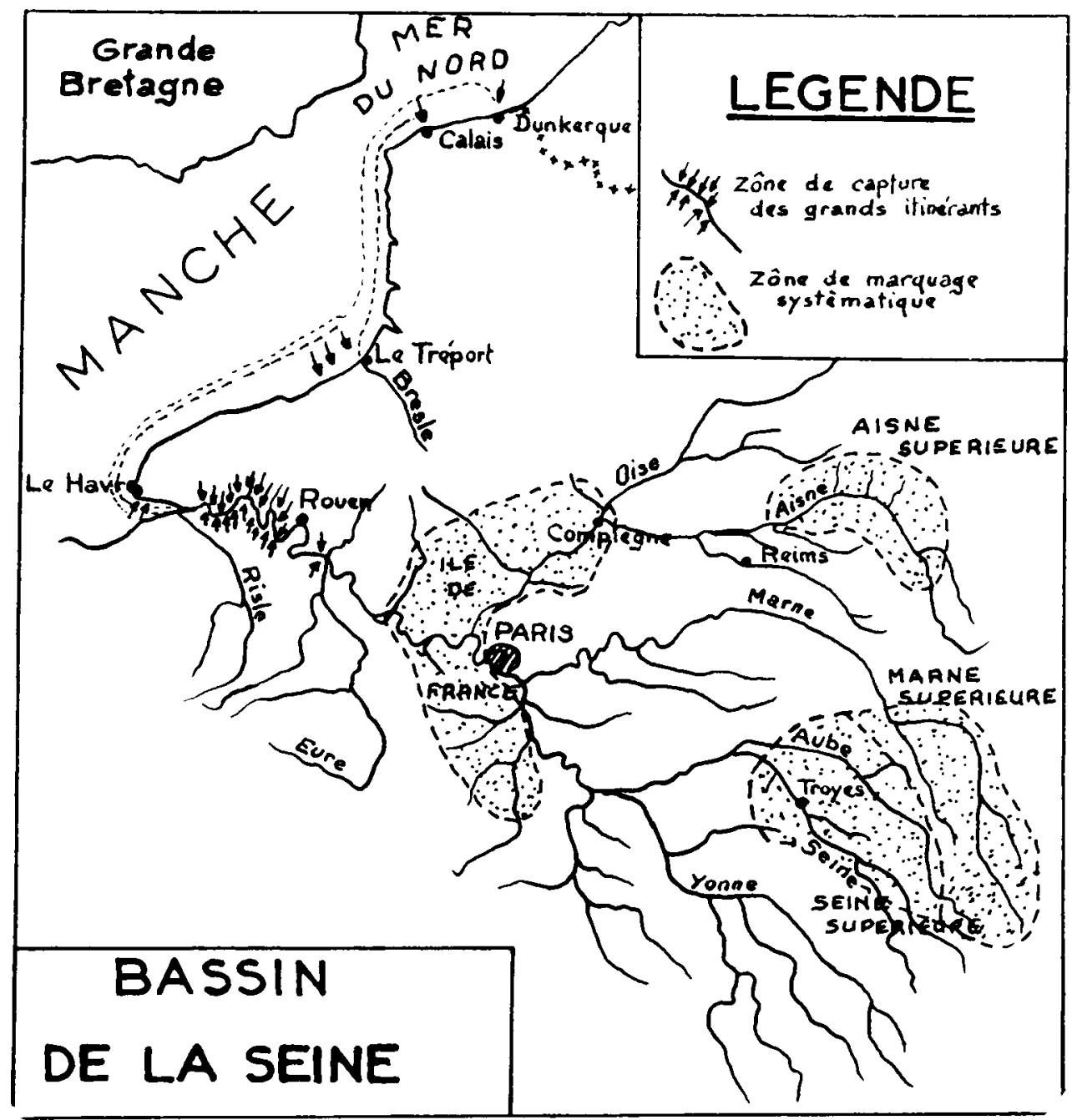

L'opération accomplie, après tranquillisation des sujets, est bien sùr complétée par la prise des dimensions et poids, par l'enregistrement de l'espèce, éventuellement du sexe et d'observations sur la morphologie, l'état sanitaire et la provenance des sujets. 
La forme actuelle des marques convient assez bien à la morphologie du maxillaire inférieur de la truite, d'autant que quatre calibres de marques permettent de tenir compte de la taille du poisson. Certaines recherches entreprises par la Station I. N. R. A. d'Hydrobiologie Continentale de Biarritz (6) portent sur des modifications de forme (marque "anneau ").

Le prix de ces marques est assez modique pour que les collectivités de pêcheurs puissent mettre en œuvre le marquage d'un nombre de sujets assez important afin que le taux de capture soit éloquent.

En principe, une collectivité détermine le nombre de sujets qu'elle peut faire marquer annuellement en fonction du taux de la prime qu'elle octroie pour tout retour de marque et de renseignements s'y rapportant, en fonction des crédits qu'elle consent pour cette opération et en fonction du taux probable de retour de marques, estimé au préalable par des opérations-tests, ce qui se traduit par la formule (2) :

$$
\mathrm{X}=\frac{\mathrm{D} \times 100}{\mathrm{X}^{\prime} \times d}
$$

où :

$X=$ nombre de sujets que l'on se propose de marquer;

$\mathrm{D}=$ montant des crédits prévus pour les retours de marques;

$d=$ montant de la récompense à l'unité de capture (prime); $\mathrm{X}^{\prime} \%=$ taux probable de retours de marques.

Dans certains cas très particuliers, les techniciens de la Région Piscicole ont marqué des sujets ayant une livrée de migrateurs, ceci au moyen de marques dites "fanion".

Il s'agit d'une plaquette, très fine, enrobée de matière plastique, numérotée, donnant l'adresse de la Station I. N. R. A. d'Hydrobiologie Continentale de Biarritz, percée d'un trou dans lequel passe un fil chirurgical dont une extrémité est enfilée sous la première nageoire dorsale de l'animal, à l'aide d'une aiguille à injection hypodermique. Cette plaquette se présente donc sous la forme d'un fanion flottant à l'extrémité d'un étrier qui passe sous la dorsale.

L'avantage de ce procédé réside dans le fait que l'animal, s'il est migrateur, peut croître en mer d'une façon considérable sans que cette croissance puisse affecter la marque, au contraire de ce qui se passerait s'il avait reçu une marque " mâchoire ".

En fait, le marquage par marque mâchoire est précieux pour suivre les dévalaisons potamiques, le marquage par marque fanion permet quant à lui de déceler les tentatives de "homing " après un séjour en mer plus ou moins long. 


\section{3. - LES EGHANTHLONNAGES}

Les échantillonnages ont porté sur des salmonidés se rassemblant chaque année en mars-avril au confluent des petits tributaires de la

Basse Seine, ainsi qu'aux abords de toutes les sources rhéocrènes entre Bonnières et Tancarville.

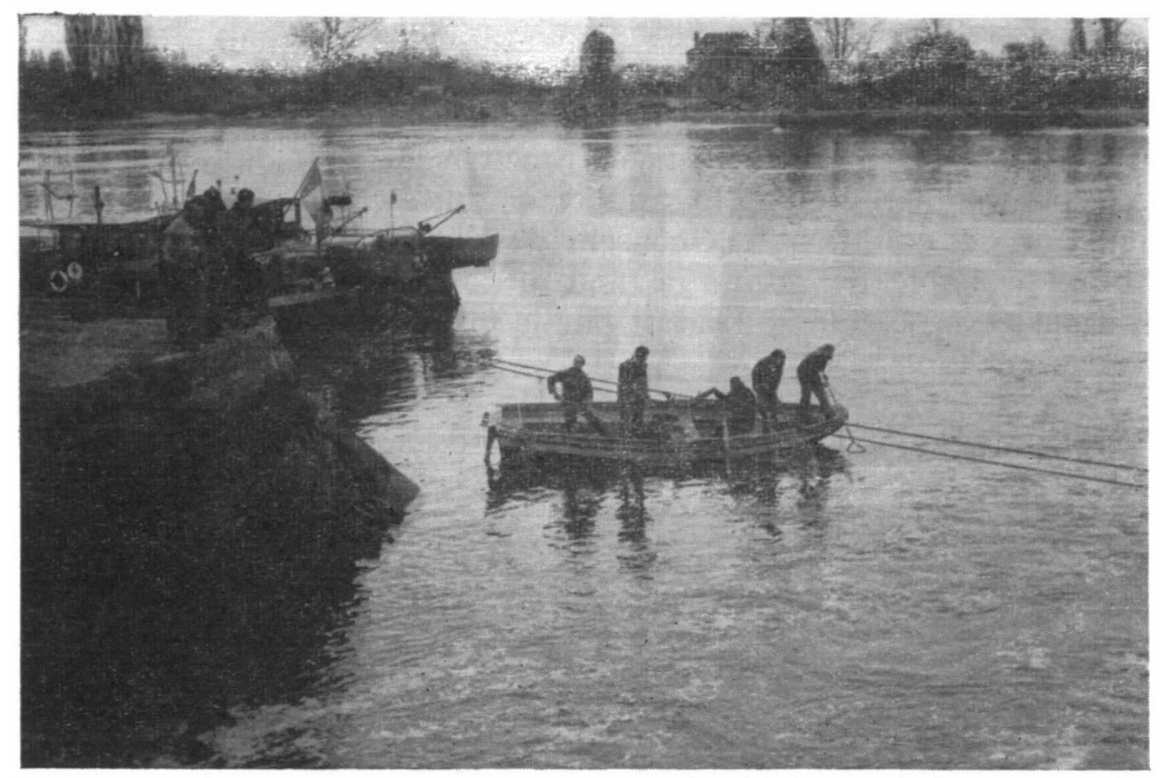

Contrôle de Salmonides en Basse-Seine par pêche électrique au confluent de la Seine et de l'Austreberthe, à Duclair (Seine-Maritime)

Ces échantillonnages ont été effectués par pêche électrique, notamment à Duclair, au confluent de l'Austreberthe, près de Caudebec, au confluent du Rançon et à Port-Jumièges sur la rive gauche de la Seine.

La Fédération Départementale des A. P. P. de la Seine-Maritime a organisé, par ailleurs, des pêches exceptionnelles à la ligne avec stockage des sujets pêchés, à la Pisciculture Fédérale de Rouen, puis à celle de Sainte-Gertrude-Maulevrier.

\section{III. - RÉSULTATS \\ DE LA GAMPAGNE DE MARQUAGE}

\section{0. - IMPORTANGE DES MARQUAGES}

Dans son état actuel, le Fichier Régional fait apparaître qu'il a été marqué 30.030 truites et truitelles dans le seul bassin de la Seine, soit par année : 


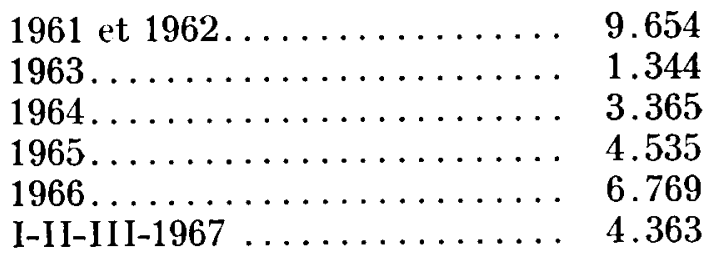

Soit, par départements :

Ardennes .............. 1.066

Haute-Marne . . . . . . . . . 8.800

Aisne ................ 144

Marne ............................ 769

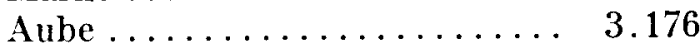

Oise $\ldots \ldots \ldots \ldots \ldots \ldots \ldots \ldots \ldots \ldots \ldots \ldots$

Seine-et-Marne............ 926

Essonnes, Val d'Oise, Yvelines. . 8.946

Eure-et-Loir .............. 409

Eure ................ 1.229

Seine-Maritime . . . . . . . . . 2.799

Les marquages systématiques et planifiés concernant les quatre grandes régions hydrographiques se sont répartis comme suit :

TABLEAU I

\begin{tabular}{|c|c|c|c|c|}
\hline Année & $\begin{array}{c}\text { Marne } \\
\text { Supérieure }\end{array}$ & $\begin{array}{c}\text { Seine } \\
\text { et Aube } \\
\text { Supérieures }\end{array}$ & $\begin{array}{c}\text { Aisne } \\
\text { Supérieure }\end{array}$ & $\begin{array}{l}\text { Seine } \\
\text { Moyenne }\end{array}$ \\
\hline $1961-1962 \ldots$ & $\ldots$ & -- & -- & 8.952 \\
\hline 1963. & 6.18 & 100 & - & - \\
\hline 196.4 & 2.178 & 430 & 40 & 207 \\
\hline 1965 & 1.970 & 789 & 172 & 350 \\
\hline $1966 \ldots \ldots \ldots$ & 3.158 & 1.407 & 436 & 416 \\
\hline I-I I-I II-1937. & 870 & 450 & 118 & 1.535 \\
\hline Total... & 8.821 & 3.176 & $1.066 j$ & 11.460 \\
\hline Total général. & \multicolumn{4}{|c|}{24.526} \\
\hline
\end{tabular}

Les autres opérations de marquage ont été opérées en annexe à des interventions à caractère très particulier tels que : sondages et inventaires de population, études du comportement des espèces devant les barrages physiologiques, écrans électriques, ou après traitement au 
carbonate de calcium de cours d'eau envasés, ou bien encore dans le cadre d'investigations à caractère typiquement local.

\section{1. - IMPORTANCE DES RETOURS DE MARQUES}

Au 31 octobre 1967, 3.138 sujets marqués ont été capturés et signalés au Fichier Régional.

Sur ces 3.138 sujets retrouvés, 3.049 n'avaient pas quitté le bassin dans lequel ils avaient été déversés, l'amplitude maximale des déplacements étant de l'ordre de $20 \mathrm{~km}$. 85 furent recueillis en Basse Seine (entre Rouen et Le Havre). 3 dans la Manche, dans les parages du Tréport, 1 en mer du Nord, à la hauteur de Dunkerque. (Le sujet recueilli dans le port de Calais n'était pas originaire du bassin de la Seine, mais provenait d'un fleuve côtier de la Manche : la Bresle.)

Le pourcentage général des retours de marques dépasse $10 \%$, soit $10,45 \%$.

I e pourcentage des retours locaux est de $10,15 \%$, celui des retours lointains de $3,06 \%$.

Les chiffres sont toutefois très inférieurs à la réalité car les retours de marques d'une région pilote ne nous sont pas parvenus et ceux d'une seconde région pilote sont assez incomplets.

Par grandes régions hydrographiques, les résultats sont les suivants :

Tablati II

\begin{tabular}{|c|c|c|c|c|c|c|c|}
\hline \multirow{2}{*}{$\begin{array}{l}\text { Régions } \\
\text { pilotes }\end{array}$} & \multirow{2}{*}{$\begin{array}{c}\text { Sujets } \\
\text { marqués } \\
\text { Nombre }\end{array}$} & \multicolumn{5}{|c|}{ Marqués et retrouvés } & \multirow{2}{*}{ Taux } \\
\hline & & $\begin{array}{l}\text { Loca- } \\
\text { lement }\end{array}$ & $\begin{array}{l}\text { Basse } \\
\text { Seine }\end{array}$ & Manche & $\begin{array}{l}\text { Mer du } \\
\text { Nord }\end{array}$ & Total & \\
\hline $\begin{array}{c}\text { Marne Supé- } \\
\text { rieure .... }\end{array}$ & 8.824 & 588 & 26 & - & - & 614 & $\begin{array}{l}6,95 \% \\
2,94 \%\end{array}$ \\
\hline $\begin{array}{c}\text { Aisne Supé- } \\
\text { rieure } . . .\end{array}$ & 1.066 & 368 & 2 & - & 1 & 371 & $\begin{array}{c}34,80 \% \\
2,81 \%\end{array}$ \\
\hline $\begin{array}{l}\text { Aube et Seine } \\
\text { Supérieures }\end{array}$ & 3.176 & $?$ & 5 & 2 & $\ldots$ & $?$ & $2,20 \%$ \\
\hline Ile de France. & 11.460 & 1.475 & 52 & 1 & - & 1.528 & $\begin{array}{c}13,33 \% \\
4,71 \%\end{array}$ \\
\hline
\end{tabular}

Nota. - Les taux en o/oo concernent les retours provenant de Basse Seine, de la Manche et de la Mer du Nord. 


\section{2. - LES DÉPLACEMENTS}

Tous les salmonidés ont un caractère itinérant plus ou moins prononcé suivant les espèces, les variétés et les races, que l'on ait affaire aux truites fario ou aux truites arc-en-ciel en ce qui concerne l'objet de la préoccupation présente.

Le dépouillement des renseignements accompagnant les marquages et les retours de marques donne toute la gamme des déplacements et migrations, depuis l'amplitude extrême, de l'ordre de $800 \mathrm{~km}$, jusqu'à un sédentarisme presque absolu, de l'ordre de quelques mètres.

\section{0. - Les grands itinérants eurhyalins.}

Il s'agit de truites capturées en mer. Sur 3.138 sujets, 4 ont été retrouvés dans la Manche et dans la Mer du Nord.

Caplures aux abords du Tréport.

Les deux sujets récupérés aux abords du Tréport ont été marqués le 31 janvier 1966. L'un a été capturé le 1 er août 1966 ; l'autre, le 9 septembre 1966.

Ils faisaient partie d'un même lot de 300 truites marquées, introduites dans la Vanne, affluent de l'Yonne, à Vulaines.

Ils ont parcouru $520 \mathrm{~km}$ en eau douce.

Celui qui a été capturé au large du Tréport a effectué, en outre, $125 \mathrm{~km}$ en mer, et son compagnon, pêché face à Criel-sur-Mer, $115 \mathrm{~km}$, soit un parcours total apparent de $645 \mathrm{~km}$ pour l'un, en 181 jours ; $635 \mathrm{~km}$ pour l'autre, en 221 jours.

Les deux truites pesaient $50 \mathrm{~g}$ lors de leur introduction dans la Vanne. Lors de la capture, le premier sujet pesait $550 \mathrm{~g}$, et le deuxième $300 \mathrm{~g}$.

Les pesées sont très vraisemblablement sérieuses, vu la notabilité des pêcheurs qui ont donné les renseignements accompagnant les marques.

\section{Capture à la jetée du port de Dinkerque.}

Le sujet pêché le 11 juillet 1967, à la jetée du port de Dunkerque, a été marqué le 27 décembre 1966 et déversé dans la Saulce, affluent de l'Aisne, bassin de l'Oise et de la Seine, dans le département des Ardennes.

Il faisait partie d'un lot de 24 truites dont 18 ont été capturées sur place pendant la saison de pêche.

Cette truite a parcouru $530 \mathrm{~km}$ en eau douce et $275 \mathrm{~km}$ en mer. Soit un total de $805 \mathrm{~km}$ en 192 jours. $470 \mathrm{~g}$.

La truite avait été déversée à la taille de $340 \mathrm{~mm}$ hors tout et pesait

Lors de la capture, elle mesurait $300 \mathrm{~mm}$ de l'œil à l'échancrure de 
la queue et pesait $1.000 \mathrm{~g}$. La coloration de sa robe était blanc argenté, celle de sa chair était rose saumon.

Les renseignements concernant ce sujet ont été transmis par l'Institut Scientifique et Technique des Pêches Maritimes, Laboratoire de Boulognesur-Mer, et par l'auteur de la capture, qui l'un et l'autre ont considéré ce salmonidé comme étant un saumon.

\section{1. - Les concentrations en basse Seine.}

Le tableau II fait apparaître que 85 salmonidés marqués ont été retrouvés en Basse Scine, notamment entre Rouen et Le Havre.

17 d'entre eux furent pêchés au même endroit, en 20 jours de pêche à la ligne (1966 et 1967) lors des concentrations signalées plus avant.

Le nombre total des captures opérées pendant ces vingt jours n'a pu être établi d'une façon précise. Il a fallu enquêter parmi les pêcheurs locaux, dont la collaboration fut malaisée étant donné que les sujets pêchés n'atteignaient pas la taille légale de capture.

M. de Sauverzac, président de la Fédération Départementale des A. P. P. de la Seine-Maritime, les gardes-pêche, les agents des Ponts et Chaussćes du Bac de Jumièges nous ont assuré que les captures étaient nombreuses et faciles lors des concentrations de mars-avril. Nous avons pu nous en rendre compte en échantillonnant par pêche électrique.

Si l'on se reporte au délai moyen mis par les 85 sujets pour parcourir la distance séparant le point de déversement dans le bassin supérieur de la Seine du point de capture en Basse Seine, on peut ctablir que la dévalaison moyenne est de 75 jours. Il faut tenir compte du fait que certaines truites, récupérées au bout de 100 à 180 jours après leur déversement, ont pu séjourner au droit de l'écoulement des sources qui donnent dans la Basse Seine à l'aplomb des falaises et y prendre d'ailleurs du poids, alors que les premières ont conservé leur poids d'origine.

A ce compte, les grands itinérants eurhyalins ont vraisemblablement prospecté d'intéressantes zones trophiques marines. Ils ont dû franchir le cap de la Hève alors que leur poids voisinait encore $50 \mathrm{~g}$ et, au cours d'une pérégrination de 120 à 150 jours, c'est-à-dire de 4 à 5 mois, prendre ainsi les poids mentionnés ci-avant, la croissance étant alors de l'ordre de 300 à $500 \mathrm{~g}$ pendant ce laps de temps.

Le mystère demeure pour une truite déverséc dans l'Aube Supérieure le 21 avril 1966, au poids de $50 \mathrm{~g}$, et pêchée en Basse Seine, à proximité de La Roche-Guyon, le 19 octobre 1966, au poids de $750 \mathrm{~g}$ : ce sujet aurait pris $700 \mathrm{~g}$ en 6 mois de séjour fluvial.

Le record de vitesse de déralaison des truites prises à Duclair et provenant tant de la Haute Seine (confluent de l'Ource et de la Seine) que de l'Aujon (affluent de l'Aube Supérieure et de la Marne) s'établit à 24 jours pour une dévalaison de $535 \mathrm{~km}$, soit une moyenne quotidienne de $23.100 \mathrm{~m}$. Ce record est à rapprocher de celui des jeunes smolts (saumoneaux d'avalaison) qui est de l'ordre de 23 à $29 \mathrm{~km} /$ jour (VIBERT 19). 
32.2. - Les déplacements potamiques acycliques.

$24,12 \%$ du nombre des sujets capturés marqués dans le Bassin Supérieur de la Seine, de l'Aube, de la Marne et de l'Aisne, ont quitté les cours d'eau enrichis et ont été capturés à des distances variant de 2 à $30 \mathrm{~km}$ du point de déversement.

Il s'agit pour $6,24 \%$ de sujets faisant partie des lots dont proviennent les truites grandes migratrices. L'échelonnement chronologique des captures correspond à une progression de la dévalaison, mais la rapidité de cette dernière ne permet pas de classer les 168 truites en question dans le contingent des grands itinérants qui, introduits en rivière en décembre et en janvier, ont déjà atteint la Basse Seine aux alentours de l'ouverture de la pêche, c'est-à-dire fin mars, alors que les sujets dont il s'agit ont été capturés de mars à août à des distances plus ou moins considérables.

Il y a lieu de penser, qu'en plus des déplacements de dépressement et d'exploration, il y a abandon d'un habitat peu favorable, recherche et colonisation d'un milieu plus favorable; il y a également au moins un cas de retour vers la pisciculture d'origine.

Lors d'un enrichissement, on constate qu'il y a étalement moyen, en 6 mois, sur $800 \mathrm{~m}$ de part et d'autre du point de déversement. Certaines dévalaisons sont de l'ordre de $20 \mathrm{~km}$ au bout du même laps de temps, par contre certains sujets ont remonté les cours d'eau jusqu'à une distance maximale de $15 \mathrm{~km}$ : il n'est pas douteux que dans la majeure partie des situations que nous avons analysées, la densité de repeuplement, trop forte sans doute eu égard au calibre et aux possibilités nourricières du parcours, a très vraisemblablement incité un certain nombre de ces animaux à fuir le confinement et à rechercher des zones plus propices (étude des déplacements de 2.127 sujets marqués recapturés, dont certains exemples figurent dans les tableaux III, IV et suivants).

\section{Cas d'une fuite devant pollution.}

C'est ainsi qu'une truite fario mesurant $235 \mathrm{~mm}$ et pesant $140 \mathrm{~g}$ fut marquée et déversée le 14 janvier 1967 dans la Vaux-Aisne-Oise-Seine ; au cours des cinq mois qui suivirent, ce sujet descendit la Vaux sur $5.500 \mathrm{~m}$, et remonta dans la Draize, affluent du précédent cours d'eau, sur $3.000 \mathrm{~m}$; elle fut donc capturée en juin 1967 ayant parcouru $8.500 \mathrm{~m}$, certainement rapidement puisqu'elle a eu le temps de profiter d'un environnement très favorable dans la Draize : de $235 \mathrm{~mm}$ elle est passée à une taille de $280 \mathrm{~mm}$, et de $140 \mathrm{~g}$ à un poids de $255 \mathrm{~g}$.

Les observations concernant la Vaux, milieu récepteur, indiquent que ce cours d'eau est classé en $2^{\mathrm{e}}$ catégorie à l'endroit du déversement : il s'agit d'une rivicre cyprinicole, souffrant au surplus d'une pollution organique (laiterie ct porcherie) provoquant sporadiquement un abondant développement de Leptomitus et de Sphaerotilus. 
La Draize, milieu colonisé, possèle par contre des eaux de bonne qualité, exemptes de pollution; le fond est constitué de sables, graviers recouverts d'herbiers à callitriches.

\section{Cas d'un retour vers la pisciculture d'origine.}

Le second cas concerne une truite fario de $210 \mathrm{~mm}$, poids $100 \mathrm{~g}$, qui, déversée le 9 novembre 1965 très en amont dans le Plumion-Vaux-AisneOise-Seine est descendue jusqu'au confluent et a remonté la Vaux jusqu'à sa pisciculture d'origine (Pisciculture Lassaux, à Signy-l'Abbaye), à proximité de laquelle elle fut capturée le 11 juin 1966, c'est-à-dire 6 mois après son introduction : le déplacement a été de $30 \mathrm{~km}=15 \mathrm{~km}$ d'avalaison dans le Plumion et $15 \mathrm{~km}$ d'amontaison dans la Vaux. Elle a donc franchi le seuil de pollution qui avait motivé la fuite du sujet dont il a été parlé précédemment.

\section{3. - Les déplacements potamiques cycliques.}

La période de reproduction provoque toujours l'apparition d'un phénomène de migration qui se traduit par la prise d'une livrée très riche et très colorée chez le mâle, encore plus chez la truite arc-en-ciel que chez la truite fario. Nous avons pu observer après dissection de reproducteurs sacrifiés, puis par lavage de l'estomac de sujets vivants mais tranquillisés (37 sujets examinés) que les animaux gras en fin d'automne ont l'estomac vide de toute proie dès que la motivation sexuelle a déclenché le phénomène de déplacement (1964 et 1965, La Semoigne-Marne-Seine, Station domaniale de Pisciculture de Neuville Le Matz-Oise-Seine). Il semble y avoir jeûne et, peut-être comme chez le saumon (8), jeùne synchonique, la synchonie étant la transformation de certaines substances, leur transfert et leur réemploi dans d'autres organes (MisLin).

Le fichier situe le tout début de la migration aux alentours du 15 aoùt avec une progression constante du phénomène.

La suspension des sources de renseignements, c'est-à-dire la fermeture de la saison de pêche, ne permet pas de donner une représentation graphique de cette progression dont l'allure est tributaire des conditions atmosphériques : pluies, ruissellement et crues, ainsi que brusques variations de température.

Les contrôles par pêche électrique montrent que les frayères sont peuplées parfois dès le mois d'octobre par les plus petits sujets (adultes vraisemblablement âgés de 3 ans, qui en sont donc à leur première fraye). Dans un cas extrême, il nous est arrivé de trouver des femelles fario, parfaitement cuvées à 20 mois (taille moyenne : $218 \mathrm{~mm}, 7$ sujets; rû du Rhône-Matz-Oise-Seine, 21 octobre 1966).

Les retours de marques en provenance de l'Ource Seine (Supérieure), complétant les études faites par inventaire démographique en 1963 et 1964, montrent que les géniteurs reprennent possession de leur territoire de chasse, voire d'une tenue très précise (au mètre près) dès la fraye 
terminée (recapture en date du 6 mars 1966). L'amplitude maximale de la migration atteint $15 \mathrm{~km}$.

Le phénomène se limite parfois à un parcours de quelques dizaines de mètres, ce qui a été noté dans la Marne Supérieure, dans l'Aisne Supérieure et dans le Grand Morin-Marne-Seine : les géniteurs, le moment venu, quittent leur territoire du cours d'eau principal et trourent leurs frayères dans un petit tributaire voisin, puis, après la ponte, le quittent pour regagner leur territoire. Cette proximité peut s'expliquer par le fait que l'apport du ruisseau améliorant les qualités de l'eau d'un cours d'eau principal, parfois tout juste salmonicole, fixe les populations de truites à son voisinage immédiat.

(A suivre.) 